\title{
Une version quantitative du théorème de Lindemann-Weierstrass
}

\author{
par
}

\author{
Mohammed Ably (Lille)
}

1. Introduction. En 1873, Hermite démontre la transcendance de $e$. Développant la méthode d'Hermite, Lindemann démontre en 1882 la transcendance de $\pi$ et énonce un théorème, contenant les résultats précédents, dont la démonstration a été complétée par Weierstrass. Ce théorème, appelé théorème de Lindemann-Weierstrass, est le suivant : Si $y_{1}, \ldots, y_{n}$ sont des nombres algébriques linéairement indépendants sur $\mathbb{Q}$ alors $e^{y_{1}}, \ldots, e^{y_{n}}$ sont algébriquement indépendants.

Dans l'étude de l'indépendance algébrique, parallèlement à l'aspect qualitatif, on s'intéresse à l'aspect quantitatif des résultats. Pour décrire l'analogue quantitatif du théorème précédent, on utilise la notion de "mesure d'indépendance algébrique" de $e^{y_{1}}, \ldots, e^{y_{n}}$; c'est la fonction de $H$ et $D$ définie par $\Phi\left(e^{y_{1}}, \ldots, e^{y_{n}}, H, D\right)=\min \left|P\left(e^{y_{1}}, \ldots, e^{y_{n}}\right)\right|$, où le minimum est pris sur l'ensemble des polynômes non nuls de $\mathbb{Z}\left[X_{1}, \ldots, X_{n}\right]$ de degré (total) $\leq D$ et de hauteur "naïve" $\leq H$; la hauteur "naïve" d'un polynôme de $\mathbb{Z}\left[X_{1}, \ldots, X_{n}\right]$ étant le maximum des valeurs absolues de ses coefficients.

En 1932, par la méthode de Siegel, K. Mahler [M] obtient une minoration asymptotique (en $H)$ de $\Phi\left(e^{y_{1}}, \ldots, e^{y_{n}}, D, H\right)$. Il démontre, sous les conditions du théorème de Lindemann-Weierstrass, que $\Phi\left(e^{y_{1}}, \ldots, e^{y_{n}}, D, H\right) \geq$ $H^{-c D^{n}}$ pour $H \geq H_{0}, H_{0}$ dépendant de $D, y_{1}, \ldots, y_{n}$ et $c=c\left(y_{1}, \ldots, y_{n}\right)$ $>0$.

Dans ce résultat, la mesure $\Phi$ est "presque" optimale; en effet, à l'aide du principe des tiroirs de Dirichlet, on montre que pour tout $n$-uplet de nombres complexes $\theta_{1}, \ldots, \theta_{n}$, il existe $D_{0}=D_{0}\left(\theta_{1}, \ldots, \theta_{n}\right)>0$ tel que pour tout $D, D \geq D_{0}$, et tout $H \geq 1$, on ait

$$
\Phi\left(\theta_{1}, \ldots, \theta_{n}, H, D\right) \leq H^{-D^{n} /(3 n !)} .
$$

Mais la dépendance de $H_{0}$ en fonction de $D$ dans le résultat de Mahler n'est pas explicite. 
En 1977, Y. Nesterenko $\left[\mathrm{N}_{1}\right]$ comble cette lacune en utilisant la méthode de Siegel sur les $E$-fonctions; sous les hypothèses du théorème de LindemannWeierstrass, il montre qu'il existe $C=C\left(y_{1}, \ldots, y_{n}\right)>0$ tel que, si l'inégalité

$$
\log \log H>C D^{2 n} \log (D+1)
$$

est vérifiée, alors on a

$$
\Phi\left(e^{y_{1}}, \ldots, e^{y_{n}}, D, H\right) \geq H^{-c_{1} D^{n}},
$$

où

$$
\begin{aligned}
c_{1}=4^{n}\left(\left[\mathbb{Q}\left(y_{1}, \ldots, y_{n}\right): \mathbb{Q}\right]\right)^{n}\left(n\left(\left[\mathbb{Q}\left(y_{1}, \ldots, y_{n}\right): \mathbb{Q}\right]\right)^{2}\right. \\
\left.+\left[\mathbb{Q}\left(y_{1}, \ldots, y_{n}\right): \mathbb{Q}\right]+1\right) .
\end{aligned}
$$

L'objet de ce texte est de raffiner ce résultat; nous démontrons le théorème suivant :

ThÉORÈme. Soient $y_{1}, \ldots, y_{n}$ des nombres algébriques $\mathbb{Q}$-linéairement indépendants et $\mathbb{K}=\mathbb{Q}\left(y_{1}, \ldots, y_{n}\right)$. Alors, il existe $C=C\left(y_{1}, \ldots, y_{n}\right)>0$ tel que pour tout polynôme $P \in \mathbb{Z}\left[X_{1}, \ldots, X_{n}\right]$ non nul, de degré $\leq D$ et de hauteur naïve $\leq H$, on ait

$$
\log \left|P\left(e^{y_{1}}, \ldots, e^{y_{n}}\right)\right| \geq-c_{2} D^{n}\left(\log H+\exp \left(C D^{n} \log (D+1)\right)\right)
$$

où

$$
c_{2}=2^{n\left(4 n^{2}+18 n+25\right)+4} n^{n^{2}+n+2}(4[\mathbb{K}: \mathbb{Q}]+n+1)^{n+2}([\mathbb{K}: \mathbb{Q}])^{n^{2}+n+1} .
$$

En particulier, si l'inégalité

$$
\log \log H>C D^{n} \log (D+1)
$$

est vérifiée, alors on a $\Phi\left(e^{y_{1}}, \ldots, e^{y_{n}}, D, H\right) \geq H^{-2 c_{2} D^{n}}$.

La condition $(* *)$ est plus faible que la condition $(*)$ dans le résultat de Nesterenko mais la constante $c_{2}$ dans notre résultat est moins bonne que la constante $c_{1}$.

La méthode utilisée par Nesterenko est la méthode de Siegel [S] sur les $E$-fonctions, alors que la méthode que nous utilisons est la méthode de Gel'fond. Le développement récent de cette méthode est basé sur des outils géométrico-algébriques tels que les lemmes de zéros (cf. $\left[\mathrm{P}_{2}\right]$, par exemple) et les critères d'indépendance algébrique. Le critère le plus général est le critère démontré par $\mathrm{P}$. Philippon dans $\left[\mathrm{P}_{1}\right]$ grâce aux techniques de l'élimination projective introduite par $\mathrm{Y}$. Nesterenko dans $\left[\mathrm{N}_{1}\right]$ pour l'étude des nombres transcendants.

Enfin, signalons que dans ce cadre, T. Töpfer [T] a obtenu récemment, en utilisant une méthode basée sur l'élimination linéaire, un résultat moins fort que le théorème ci-dessus. 
I. Notations et résultats préliminaires. Pour un polynôme $P$ à coefficients $p_{1}, \ldots, p_{l}$ dans un corps de nombres $\mathbb{F}$, on désigne par $h(P)$ la hauteur logarithmique de Weil du point $\left(1, p_{1}, \ldots, p_{l}\right)$ de $\mathbb{P}_{l}(\mathbb{F})$ (cf. $\left[\mathrm{Wa}_{1}\right.$ ], p. 19) et par $\operatorname{deg} P$ le degré total de $P$.

Si $P$ est à coefficients entiers rationnels non tous nuls, on a $h(P)=$ $\log H(P)$, où $H(P)$ est la hauteur naïve de $P$.

Pour un idéal $I$ pur de $\mathbb{F}\left[X_{1}, \ldots, X_{n}\right]$ et $\underline{\omega} \in \mathbb{C}^{n}, \operatorname{ht}(I)$, deg $I$ et $\|I\|_{\underline{\omega}}$ désignent respectivement la hauteur, degré et valeur absolue de $I$ en $\underline{\omega}$ définis via les formes $U$-éliminantes associées à $I$ (cf. $\left.\left[\mathrm{P}_{1}\right]\right)$.

Pour démontrer des résultats qualitatifs d'indépendance algébrique de plusieurs nombres, le critère d'indépendance algébrique le plus efficace est le critère de $\mathrm{P}$. Philippon (cf. $\left.\left[\mathrm{P}_{1}\right]\right)$. La version quantitative de ce critère qui permet d'obtenir des mesures d'indépendance algébrique a été démontrée par E. M. Jabbouri (cf. [J]). On en déduit l'énoncé suivant qui permet d'avoir des mesures d'indépendance algébrique en codimension un.

CRITÈre. Soient $\mathbb{K}$ un corps de nombres et $\underline{\theta}=\left(\theta_{1}, \ldots, \theta_{n}\right) \in \mathbb{C}^{n}$. Soient $1 \leq \delta, \tau, \sigma$ et $U$ des nombres réels positifs avec $\sigma \geq 1$ et $U \geq 2 \max \left(\tau, \sigma^{n}\right)$. On suppose que pour tout entier $S$ vérifiant

(a) $\tau / \sigma^{n}<S \leq U / \sigma^{n}$,

il existe une famille finie de polynômes $\left(Q_{S, j}\right)_{j=1, \ldots, m(S)} \subset \mathbb{K}\left[X_{1}, \ldots, X_{n}\right]$ telle que :

(b) $\max _{j} \operatorname{deg} Q_{S, j} \leq \delta$;

(c) $\max _{j} h\left(Q_{S, j}\right)+\delta(n+1) \log (n+1) \leq \tau$;

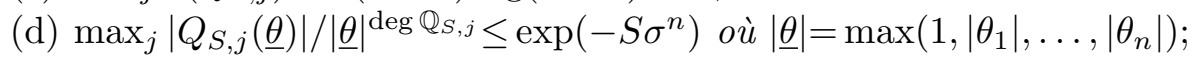

(e) les polynômes $Q_{S, j}(j=1, \ldots, m(S))$ sont sans zéros communs dans la boule de centre $\underline{\theta}$ et de rayon $\exp \left(-S \sigma^{n+1}\right)$ de $\mathbb{C}^{n}$.

Alors, pour tout polynôme $P$ non nul de $\mathbb{Z}\left[X_{1}, \ldots, X_{n}\right]$, de hauteur logarithmique $h$ et de degré $D$, satisfaisant

(f) $(4[\mathbb{K}: \mathbb{Q}]+n+1)(27 \sigma)^{n}\left(h \delta^{n}+\left(\tau n+\delta(n+1)^{2} \log (n+1)\right) D \delta^{n-1}\right) \leq U$, on $a$

$\log \left|P\left(\theta_{1}, \ldots, \theta_{n}\right)\right| \geq-U-n D \log 2(n+1)-n^{2} \log |\underline{\theta}|-2 n \log (n+1)$.

Preuve. Remarquons d'abord que la hauteur utilisée dans le critère de Jabbouri (cf. $[\mathrm{J}]$ ) est la hauteur invariante $\bar{h}$ définie dans $[\mathrm{J}]$. Nous l'avons remplacé dans l'énoncé précédent par la hauteur logarithmique absolue $h$ (cela revient à remplacer, localement en les places archimédiennes, la mesure de Mahler par la hauteur naïve) en utilisant l'estimation

$$
\bar{h}(P) \leq h(P)+(\log (n+1)) \operatorname{deg} P .
$$


Cela explique les légères modifications intervenues dans les propriétés (c) et (f) par rapport à l'énoncé original de Jabbouri.

Notons encore $\underline{\theta}$ le point de $\mathbb{P}_{n}(\mathbb{C})$ de coordonnées $\left(1, \theta_{1}, \ldots, \theta_{n}\right)$, h $P$ l'homogénéisé de $P$ dans $\mathbb{Z}\left[X_{0}, \ldots, X_{n}\right]$ et $\mathcal{I}$ l'idéal de $\mathbb{Z}\left[X_{0}, \ldots, X_{n}\right]$ engendré par ${ }^{\mathrm{h}} P$.

Pour un polynôme $Q \in \mathbb{C}\left[X_{1}, \ldots, X_{n}\right]$, on note $H(Q)$ (resp. $\left.M(Q)\right)$ la hauteur naïve (resp. la mesure de Mahler de $Q$ ). On utilisera les inégalités suivantes :

$$
H(Q) 2^{-\operatorname{deg} Q} \leq M(Q) \leq H(Q)(n+1)^{\operatorname{deg} Q} .
$$

Soient $u_{i, j}, 0 \leq i \leq n-1,0 \leq j \leq n$, des nouvelles variables; on pose $u_{i}=\left(u_{i, j}\right)_{0 \leq j \leq n}(i=0, \ldots, n-1)$. Une forme $U$-éliminante de ${ }^{\mathrm{h}} \mathcal{I}$ (cf. $\left[\mathrm{N}_{2}\right]$, lemme 2) est la forme $f$ de $\mathbb{Z}\left[u_{0}, \ldots, u_{n-1}\right]$ définie par

$$
f\left(u_{0}, \ldots, u_{n-1}\right)={ }^{\mathrm{h}} P\left(\Delta_{0}, \ldots, \Delta_{n}\right)
$$

où $\Delta_{j}$ est le déterminant du cofacteur de $X_{j}$ dans la matrice

$$
\left(\begin{array}{ccc}
X_{0} & \ldots & X_{n} \\
u_{0,0} & \ldots & u_{0, n} \\
\ldots \ldots & \ldots & \ldots \\
u_{n-1,0} & \ldots & u_{n-1, n}
\end{array}\right) .
$$

On a par définition (cf. $\left[\mathrm{P}_{1}\right]$, définition 1.14 avec $\left.\underline{d}=\underline{1}\right) \operatorname{deg} \mathcal{I}=\operatorname{deg} f$. Comme $\operatorname{deg} f=n \operatorname{deg} P$, on a $\operatorname{deg} \mathcal{I}=n \operatorname{deg} P$. Puisque $f \in \mathbb{Z}\left[u_{0}, \ldots, u_{n-1}\right]$, on a $\operatorname{ht}(\mathcal{I})=\log M(f)$. Comme

$$
M(f) \leq H(f)(n+1)^{\operatorname{deg} f} \quad \text { et } \quad H(f) \leq H(P)((n+1) !)^{\operatorname{deg} P},
$$

on en déduit que

$$
\operatorname{ht}(\mathcal{I}) \leq \log H(P)+(2 n+1) \log (n+1) \operatorname{deg} P .
$$

Soient $S^{(i)}=\left(s_{k, l}^{(i)}\right)_{\substack{0 \leq k \leq n \\ 0 \leq l \leq n}}(i=0, \ldots, n-1)$ des matrices antisymétriques génériques d'ordre $n+1$ (les variables $s_{k, l}^{(i)}(0 \leq k \leq n, 0 \leq l \leq n)$ sont liées algébriquement par les seules relations $s_{k, k}^{(i)}=0$ et $s_{k, l}^{(i)}+s_{l, k}^{(i)}=0$ si $l \neq k$ ). On note

$$
S^{(i)} \cdot \underline{\theta}=\left(\sum_{k=0}^{n} s_{k, l}^{(i)} \theta_{k}\right)_{0 \leq l \leq n},
$$

$\partial_{\theta}(f)\left(S^{(0)}, \ldots, S^{(n-1)}\right)$ le polynôme de $\mathbb{C}\left[S^{(0)}, \ldots, S^{(n-1)}\right]$ défini par

$$
\partial_{\theta}(f)\left(S^{(0)}, \ldots, S^{(n-1)}\right)=f\left(S^{(0)} \cdot \underline{\theta}, \ldots, S^{(n-1)} \cdot \underline{\theta}\right)
$$

et $L_{\theta}\left(X_{0}, \ldots, X_{n}\right)$ la forme linéaire définie par $L_{\theta}(\underline{X})=\sum_{j=0}^{n} X_{j} \theta_{j}$.

D'après la définition 1.15 de $\left[\mathrm{P}_{1}\right]$,

$$
\|\mathcal{I}\|_{\underline{\theta}}=: \frac{M\left(\partial_{\theta}(f)\right)}{M(f)\left(M\left(L_{\theta}\right)\right)^{\operatorname{deg} f}} .
$$


On a

$$
M\left(\partial_{\theta}(f)\right) \leq H\left(\partial_{\theta}(f)\right)(n+1)^{\operatorname{deg} \partial_{\theta}(f)} \leq H\left(\partial_{\theta}(f)\right)(n+1)^{\operatorname{deg} f}
$$

et (cf. $\left[\mathrm{N}_{2}\right]$, preuve de la proposition 1 ) on a l'estimation

$$
H\left(\partial_{\theta}(f)\right) \leq\left|P\left(\theta_{1}, \ldots, \theta_{n}\right)\right|(n+1)^{2 n^{2}}|\underline{\theta}|^{n^{2}-n} .
$$

D'autre part,

$$
M(f) \geq H(f) 2^{-\operatorname{deg} f} \geq 2^{-\operatorname{deg} f} \geq 2^{-n \operatorname{deg} P}
$$

et

d'où

$$
M\left(L_{\theta}\right) \geq \frac{H\left(L_{\theta}\right)}{2} \geq \frac{1}{2}|\underline{\theta}|,
$$

$$
\|\mathcal{I}\|_{\underline{\theta}} \leq\left|P\left(\theta_{1}, \ldots, \theta_{n}\right)\right| 2^{n \operatorname{deg} P}(n+1)^{n(\operatorname{deg} P+2 n)}|\underline{\theta}|^{n^{2}-n(\operatorname{deg} P+1)} .
$$

Les conditions du critère de Jabbouri (cf. $[\mathrm{J}]$ ) étant vérifiées avec $k=$ $n-1$, l'idéal $\mathcal{I}$ étant de dimension $n-1$, d'après ce même critère on a $\log \|\mathcal{I}\|_{\underline{\theta}} \geq-U$. Cette inégalité jointe à l'inégalité précédente entraîne le critère précédent.

Pour des nombres réels $c>0, L \geq e$ et $0<\nu<1$, on définit les fonctions positives suivantes :

$\delta_{c}(L, \nu)=c(\log L)^{\nu}, \quad H_{c}(L, \nu)=c L(\log L)^{1-\nu} \quad$ et $\quad r_{c}(L)=c L \log L$.

On considère, pour toute la suite, $y_{1}, \ldots, y_{n}, n$ nombres algébriques $\mathbb{Q}$ linéairement indépendants. Notons $\mathbb{K}=\mathbb{Q}\left(y_{1}, \ldots, y_{n}\right), \theta_{i}=e^{y_{i}}(i=$ $1, \ldots, n), \underline{\theta}=\left(\theta_{1}, \ldots, \theta_{n}\right)$, den $\left(y_{i}\right)$ le dénominateur de $y_{i}$ et $y_{i}$ le maximum des valeurs absolues des $\mathbb{Q}$ conjugués de $y_{i}$ dans $\mathbb{C}$.

La démonstration du théorème est basée sur la proposition principale qui fait l'objet de la section suivante.

II. La proposition principale. L'objet de cette proposition est de construire une famille de polynômes vérifiant les conditions du critère précédent.

Proposition PRINCIPALE. Il existe un nombre réel $L_{0}>0$, ne dépendant que de $y_{1}, \ldots, y_{n}$, tel que pour tout $L \in \mathbb{R}, L \geq L_{0}$, et tout $\nu \in \mathbb{R}$, $0<\nu<1$, vérifiant les conditions (1), (2) et (3) suivantes:

(1) $\log \log L \geq 2(n+7) \log 2[\mathbb{K}: \mathbb{Q}] / \nu$,

(2) $(\log L)^{1-\nu} \geq c_{3}$, avec

$$
c_{3}=2^{n+5}[\mathbb{K}: \mathbb{Q}]\left(\log \max _{1 \leq i \leq n}\left(\left|y_{i}\right|+1\right)+\log n+\log \max _{1 \leq i \leq n}\left(\operatorname{den}\left(y_{i}\right)\right)\right)
$$

(3) $8[\mathbb{K}: \mathbb{Q}] \nu(\log L)^{\nu} \leq \log L / \log \log L$,

il existe un idéal $I_{L, \nu}$ engendré par des polynômes $G_{L, \nu, j}\left(1 \leq j \leq m^{\prime}(L, \nu)\right)$ de $\mathbb{K}\left[X_{1}, \ldots, X_{n}\right]$ satisfaisant les propriétés suivantes: 
(i) $I_{L, \nu}$ n'a pas de zéros dans la boule de $\mathbb{C}^{n}$ de centre $\underline{\theta}$ et de rayon $\exp \left(-r_{1}(L)\right)$

(ii) $\max _{1 \leq j \leq m^{\prime}(L, \nu)}\left|G_{L, \nu, j}(\underline{\theta})\right| \leq \exp \left(-r_{1 / 2}(L)\right)$,

(iii) $\max _{1 \leq j \leq m^{\prime}(L, \nu)} \operatorname{deg} G_{L, \nu, j} \leq \delta_{c_{4}}(L, \nu)$,

$$
\begin{aligned}
& \max _{1 \leq j \leq m^{\prime}(L, \nu)} h\left(G_{L, \nu, j}\right) \leq H_{c_{5}}(L, \nu) \text {, où } \\
& \quad c_{4}=2^{2 n+7}[\mathbb{K}: \mathbb{Q}] n \quad \text { et } \quad c_{5}=2^{2 n+10}[\mathbb{K}: \mathbb{Q}]+2 .
\end{aligned}
$$

Le schéma de démonstration de cette proposition est une variante du schéma classique de démonstration de transcendance par la méthode de Gel'fond.

La démonstration se fait en 3 pas :

1 er pas. Construction des "polynômes auxiliaires". Pour $\underline{h}=\left(h_{1}, \ldots, h_{n}\right)$ $\in \mathbb{N}^{n}, \underline{l}=\left(l_{1}, \ldots, l_{n}\right) \in \mathbb{N}^{n}, \underline{i}=\left(i_{1}, \ldots, i_{n}\right) \in \mathbb{N}^{n}, P \in \mathbb{K}\left[X_{1}, \ldots, X_{n}\right]$, $\beta \in \mathbb{N}, \gamma \in \mathbb{N}$ et $s \in \mathbb{N}$, on note

$$
\begin{gathered}
\underline{h} \cdot \underline{y}=h_{1} y_{1}+\ldots+h_{n} y_{n}, \quad \underline{h}+\underline{l}=\left(h_{1}+l_{1}, \ldots, h_{n}+l_{n}\right), \\
|\underline{h}|=\max _{1 \leq j \leq n}\left|h_{j}\right|, \quad\|\underline{h}\|=h_{1}+\ldots+h_{n}, \\
D^{i} P=\frac{1}{i_{1} ! \ldots i_{n} !} \partial_{X_{1}}^{i_{1}} \ldots \partial_{X_{n}}^{i_{n}} P, \quad \mathcal{M}_{\beta, \gamma}(Z, X)=Z^{\beta} X^{\gamma}, \\
\Delta_{s} \mathcal{M}_{\beta, \gamma}(Z, X)=\sum_{s^{\prime}=0}^{\min (\beta, s)}\left(\begin{array}{c}
s \\
s^{\prime}
\end{array}\right) \beta(\beta-1) \ldots\left(\beta-s^{\prime}+1\right) \gamma^{s-s^{\prime}} Z^{\beta-s^{\prime}} X^{\gamma} .
\end{gathered}
$$

Notons que

$$
\frac{d^{s}}{d z^{s}} \mathcal{M}_{\beta, \gamma}\left(z, e^{z}\right)=\Delta_{s} \mathcal{M}_{\beta, \gamma}\left(z, e^{z}\right) .
$$

Enfin, pour $\underline{Y}=\left(Y_{1}, \ldots, Y_{n}\right)$, on pose

$$
\mathcal{M}_{\beta, \gamma, \underline{h}, s}(\underline{Y})=\Delta_{s} \mathcal{M}_{\beta, \gamma}\left(\underline{h} \cdot \underline{y}, \prod_{i=1}^{n} Y_{i}^{h_{i}}\right) .
$$

Comme on a posé $\theta_{i}=e^{y_{i}}$, on a évidemment

$$
\mathcal{M}_{\beta, \gamma, \underline{h}, s}(\underline{\theta})=\Delta_{s} \mathcal{M}_{\beta, \gamma}\left(\underline{h} \cdot \underline{y}, e^{\underline{h} \cdot \underline{y}}\right) .
$$

Soit $L_{0}=L_{0}\left(y_{1}, \ldots, y_{n}\right)$ un nombre réel assez grand. Dans la suite, on désigne par $L$ un paramètre réel $\geq L_{0}$, et par $M, D$ et $T^{\prime}$ des paramètres entiers $\geq e$. On pose

$$
\begin{gathered}
\varphi_{1}\left(L, D, M, T^{\prime}\right)=T^{\prime}\left(\log L+\log D+\log T^{\prime}\right)+L \log M+n \log n D M \\
+\left([\mathbb{K}: \mathbb{Q}] \max _{1 \leq i \leq n} h\left(y_{i}\right)+\log \max _{1 \leq i \leq n} \operatorname{den}\left(y_{i}\right)+\log 2 n\right) L+\log L D \\
\varphi_{2}\left(L, D, M, T^{\prime}\right)=\varphi_{1}\left(L, D, M, T^{\prime}\right)+\left(n \log \left(\max _{i}\left|\theta_{i}\right|+1\right)+n^{2}\right) D M .
\end{gathered}
$$


LEMME 1. Si la condition

$$
L D>2^{n+1}[\mathbb{K}: \mathbb{Q}] T^{\prime} M^{n}
$$

est vérifiée, alors il existe des polynômes $P_{\beta, \gamma}(0 \leq \beta<L, 0 \leq \gamma<D)$ non tous nuls de $\mathbb{Z}[\underline{Y}]$ satisfaisant

$$
\operatorname{deg} P_{\beta, \gamma} \leq n D M, \quad h\left(P_{\beta, \gamma}\right) \leq \varphi_{1}\left(L, D, M, T^{\prime}\right)
$$

et tels que les polynômes définis par

$$
Q_{s, \underline{h}}(\underline{Y})=\sum_{\substack{0 \leq \beta<L \\ 0 \leq \gamma<D}} P_{\beta, \gamma}(\underline{Y}) \mathcal{M}_{\beta, \gamma, s, \underline{h}}(\underline{Y})
$$

soient nuls pour tout $s \in \mathbb{N}, 0 \leq s<T^{\prime}$, et tout $\underline{h} \in \mathbb{N}^{n},|\underline{h}|<M$.

Preuve. Si $|\underline{h}| \leq M$ et $\gamma \leq D$, on a $\operatorname{deg} \mathcal{M}_{\beta, \gamma, \underline{h}, s} \leq n D M$. En notant $m_{\beta, \gamma, \underline{h}, s, \underline{j}}$ les coefficients de $\mathcal{M}_{\beta, \gamma, \underline{h}, s}, \mathcal{M}_{\beta, \gamma, \underline{h}, s}$ s'écrit

$$
\mathcal{M}_{\beta, \gamma, \underline{h}, s}(\underline{Y})=\sum_{\|\underline{j}\| \leq n D M} m_{\beta, \gamma, \underline{h}, s, \underline{j}} \underline{\underline{j}} \underline{\underline{j}} .
$$

Pour $|\underline{h}| \leq M, \gamma \leq D, s \leq T^{\prime}$ et $\|\underline{j}\| \leq n D M$, on vérifie que

$$
\begin{aligned}
& \log \overline{m_{\beta, \gamma, \underline{h}, s, \underline{j}}} \\
& \quad \leq T^{\prime}\left(\log L+\log D+\log T^{\prime}\right)+L\left(\log M+\log n+\log \max _{i}\left|\bar{y}_{i}\right|\right)+\log L,
\end{aligned}
$$

où $\left\lceil m_{\beta, \gamma, s, \underline{h}, \underline{j}}\right.$ désigne le maximum de valeurs absolues des $\mathbb{Q}$-conjugués de $m_{\beta, \gamma, s, \underline{h}, \underline{j}}$ dans $\mathbb{C}$.

Soient $p_{\beta, \gamma, \underline{i}}, 0<\beta \leq L, 0 \leq \gamma<D, \underline{i}=\left(i_{1}, \ldots, i_{n}\right),\|\underline{i}\| \leq n D M$, des nouvelles variables. Notons

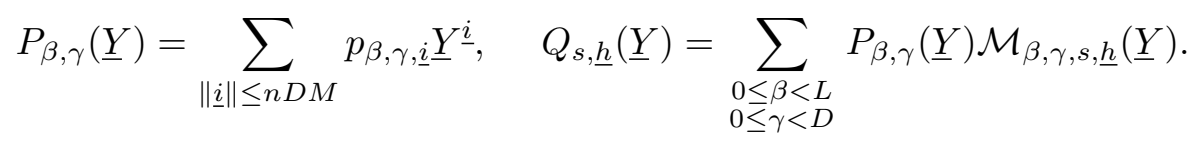

Le système $\left\{Q_{s, \underline{h}}=0: s \in \mathbb{N}, 0 \leq s<T^{\prime}, \underline{h} \in \mathbb{N}^{n},|\underline{h}|<M\right\}$ est équivalent au système linéaire, en les inconnues $p_{\beta, \gamma, \underline{i}}$ et à coefficients $m_{\beta, \gamma, s, \underline{h}, \underline{j}}$, suivant :

$$
\begin{aligned}
& \sum_{\substack{0 \leq \beta<L \\
0 \leq \gamma<D \\
\underline{i}+\underline{j}=\underline{k}}} p_{\beta, \gamma, \underline{i}} m_{\beta, \gamma, s, \underline{h}, \underline{j}}=0, \\
& \quad\|\underline{k}\| \leq 2 n D M, s \in \mathbb{N}, 0 \leq s<T^{\prime}, \underline{h} \in \mathbb{N}^{n},|\underline{h}|<M .
\end{aligned}
$$

Le nombre d'inconnues de $(\mathrm{S})$ est supérieur ou égal à $(L-1) D\left(\begin{array}{c}n D M+n-1 \\ n\end{array}\right)$ et le nombre d'équations de (S) est majoré par $T^{\prime} M^{n}\left(\begin{array}{c}2 n D M+n-1 \\ n\end{array}\right)$.

Notons $\operatorname{den}\left(y_{j}\right)$ le dénominateur de $y_{j}$ et $\delta=\max _{1 \leq j \leq n} \operatorname{den}\left(y_{j}\right)$. En multipliant par $\delta^{L}$ les deux membres du système $(\mathrm{S})$, on obtient un système 
$\left(\mathrm{S}^{\prime}\right)$ d'équations linéaires homogènes à coefficients entiers dans $\mathbb{K}$, équivalent à $(\mathrm{S})$.

Sous la condition $\left(\mathcal{C}_{1}\right)$, le lemme de Siegel (cf. $\left[\mathrm{Wa}_{2}\right]$, lemme 1.3.1) montre qu'il existe des entiers naturels non tous nuls $\left(p_{\beta, \gamma, \underline{i}}, 0 \leq \beta<L, 0 \leq \gamma<D\right.$, $\left.\underline{i} \in \mathbb{N}^{n},\|\underline{i}\| \leq n D M\right)$ vérifiant $\left(\mathrm{S}^{\prime}\right)$ et donc $(\mathrm{S})$. En plus, d'après ce lemme, on a

$$
\log \max _{\beta, \gamma, \underline{l}}\left|p_{\beta, \gamma, \underline{i}}\right| \leq \log \left(\sqrt{2} L D \delta^{L} \max _{\beta, \gamma, s, \underline{h}, \underline{j}} \mid m_{\beta, \gamma, s, \underline{h}, \underline{j}}\right) .
$$

Comme $P_{\beta, \gamma}(\underline{Y}) \in \mathbb{Z}[\underline{Y}]$, on a $h\left(P_{\beta, \gamma}\right)=\log \max _{\underline{i}}\left|p_{\beta, \gamma, \underline{i}}\right|$; ces polynômes $P_{\beta, \gamma}(0 \leq \beta<L, 0 \leq \gamma<D)$ vérifient alors le lemme 1 .

"Modification" des polynômes. Les polynômes $Q_{s, \underline{h}}\left(0 \leq s<T^{\prime},|\underline{h}|<\right.$ $M)$ peuvent posséder un zéro commun dans un voisinage de $\underline{\theta}$ pour cela nous allons les modifier, suivant une idée de G. Diaz (cf. [D]), afin d'obtenir des polynômes satisfaisant la condition (i) de la proposition 1 .

Soient $r>0$ et $\underline{\tilde{\theta}}$ dans $B\left(\underline{\theta}, e^{-r}\right)$, la boule de $\mathbb{C}^{n}$ de centre $\underline{\theta}$ et de rayon $e^{-r}$. Comme les polynômes $P_{\beta, \gamma}(0 \leq \beta<L, 0 \leq \gamma<D)$ donnés par le lemme 1 ne sont pas tous nuls et de degrés majorés (pour $D$ et $M$ fixés), il existe $j=: j(\underline{\widetilde{\theta}}) \in \mathbb{N}^{n}$ tel que les nombres $D^{j} P_{\beta, \gamma}(\underline{\widetilde{\theta}}), 0 \leq \beta<L, 0 \leq \gamma<D$, ne soient pas tous nuls et que les nombres $D^{i} P_{\beta, \gamma}(\underline{\widetilde{\theta}}), 0 \leq \beta<L, 0 \leq \gamma<D$, soient tous nuls dès que $\underline{i}$ vérifie $\|\underline{i}\|<\|\underline{j}\|$. Il suffit de prendre $\underline{j}$ tel que

$$
\|\underline{j}\|=\min \left\{\|\underline{i}\|: \exists \beta, 0 \leq \beta<L, \exists \gamma, 0 \leq \gamma \leq D, D^{\underline{i}} P_{\beta, \gamma}(\underline{\widetilde{\theta}}) \neq 0\right\} .
$$

Notons

$$
\begin{aligned}
\mathcal{I}_{r} & =\left\{\underline{j}=\underline{j}(\underline{\widetilde{\theta}}): \underline{\tilde{\theta}} \in B\left(\underline{\theta}, e^{-r}\right)\right\}, \\
Q_{s, \underline{h}, \underline{j}}(\underline{Y}) & =\sum_{\substack{0 \leq \beta<L \\
0 \leq \gamma<D}} D^{j} P_{\beta, \gamma}(\underline{Y}) \mathcal{M}_{\beta, \gamma, s, \underline{h}}(\underline{Y}) .
\end{aligned}
$$

Lemme 2. Pour tout $\underline{j} \in \mathcal{I}_{r}$, tout $s \in \mathbb{N}, 0 \leq s<T^{\prime}$, et tout $\underline{h} \in \mathbb{N}^{n}$, $|\underline{h}|<M$, on a

$$
\left|Q_{s, \underline{h}, \underline{j}}(\underline{\theta})\right| \leq \exp \left(-r+2 \varphi_{2}\left(L, D, M, T^{\prime}\right)+\log 2 L D\right) .
$$

Pre u ve. Soient $\underline{j} \in \mathcal{I}_{r}, \underline{\tilde{\theta}} \in B\left(\underline{\theta}, e^{-r}\right)$ tels que $\underline{j}=\underline{j}(\underline{\widetilde{\theta}}), s \in \mathbb{N}, 0 \leq s<$ $T^{\prime}$, et $\underline{h} \in \mathbb{N}^{n},|\underline{h}|<\bar{M}$. On a

$$
\begin{aligned}
& \left|D^{\underline{j}} P_{\beta, \gamma}(\underline{\widetilde{\theta}})-D^{j} P_{\beta, \gamma}(\underline{\theta})\right| \\
& \quad \leq \max _{i}\left|\theta_{i}-\widetilde{\theta}_{i}\right| \cdot H\left(D^{\underline{j}} P_{\beta, \gamma}\right)\left(\max _{i}\left|\theta_{i}\right|+1\right)^{\operatorname{deg}\left(D^{\underline{j}} P_{\beta, \gamma}\right)}\left(\operatorname{deg} D^{\underline{j}} P_{\beta, \gamma}+1\right)^{n}
\end{aligned}
$$

or,

$$
H\left(D^{\underline{j}} P_{\beta, \gamma}\right) \leq 2^{\operatorname{deg} P_{\beta, \gamma}} H\left(P_{\beta, \gamma}\right) \leq \exp \left(\varphi_{1}\left(L, D, M, T^{\prime}\right)\right),
$$


donc on obtient

$$
\begin{aligned}
\left|D^{j} P_{\beta, \gamma}(\underline{\widetilde{\theta}})-D^{j} P_{\beta, \gamma}(\underline{\theta})\right| & \leq \max _{i}\left|\theta_{i}-\widetilde{\theta}_{i}\right| \cdot \exp \left(\varphi_{2}\left(L, D, M, T^{\prime}\right)\right) \\
& \leq \exp \left(-r+\varphi_{2}\left(L, D, M, T^{\prime}\right)\right) .
\end{aligned}
$$

De la même façon, on a

$$
\left|\mathcal{M}_{\beta, \gamma, s, \underline{h}}(\underline{\theta})-\mathcal{M}_{\beta, \gamma, s, \underline{h}}(\underline{\widetilde{\theta}})\right| \leq \exp \left(-r+\varphi_{2}\left(L, D, M, T^{\prime}\right)\right) .
$$

Par ailleurs, on a

$$
\begin{aligned}
\left|D^{\underline{j}} P_{\beta, \gamma}(\underline{\theta})\right| & \leq H\left(D^{j} P_{\beta, \gamma}\right)\left(\max _{i}\left|\theta_{i}\right|+1\right)^{\operatorname{deg} D^{\underline{j}} P_{\beta, \gamma}}\left(\operatorname{deg} D^{\underline{j}} P_{\beta, \gamma}+1\right)^{n} \\
& \leq \exp \left(\varphi_{2}\left(L, D, M, T^{\prime}\right)\right) .
\end{aligned}
$$

De même, $\left|\mathcal{M}_{\beta, \gamma, s, \underline{h}}(\underline{\widetilde{\theta}})\right| \leq \exp \left(\varphi_{2}\left(L, D, M, T^{\prime}\right)\right)$.

Or, on a

$$
\begin{aligned}
& \left|Q_{s, \underline{h}, \underline{j}}(\underline{\theta})-Q_{s, \underline{h}, \underline{j}}(\underline{\widetilde{\theta}})\right| \leq \sum_{\substack{0 \leq \beta<L \\
0 \leq \gamma<D}}\left|D^{\underline{j}} P_{\beta, \gamma}(\underline{\theta})\right| \cdot\left|\mathcal{M}_{\beta, \gamma, s, \underline{h}}(\underline{\theta})-\mathcal{M}_{\beta, \gamma, s, \underline{h}}(\underline{\widetilde{\theta}})\right| \\
& +\sum_{\substack{0 \leq \beta<L \\
0 \leq \gamma<D}}\left|D^{\underline{j}} P_{\beta, \gamma}(\underline{\widetilde{\theta}})-D^{\underline{j}} P_{\beta, \gamma}(\underline{\theta})\right| \cdot\left|\mathcal{M}_{\beta, \gamma, s, \underline{h}}(\underline{\widetilde{\theta}})\right|,
\end{aligned}
$$

d'où

$$
\left|Q_{s, \underline{h}, \underline{j}}(\underline{\theta})-Q_{s, \underline{h}, \underline{j}}(\underline{\widetilde{\theta}})\right| \leq \exp \left(-r+2 \varphi_{2}\left(L, D, M, T^{\prime}\right)+\log 2 L D\right) .
$$

De la définition de $\underline{j}$, on tire l'égalité $Q_{s, \underline{h}, j}(\underline{\widetilde{\theta}})=\left(D^{j} Q_{s, \underline{h}}\right)(\underline{\widetilde{\theta}})$; comme $Q_{s, \underline{h}}=0$, on en déduit que $Q_{s, \underline{h}, \underline{j}}(\underline{\widetilde{\theta}})=0$ et l'inégalité précédente entraîne le lemme.

$2 e$ pas. Extrapolation. Moyennant certaines conditions sur $L, D, M, T^{\prime}$ et $r$, nous allons extrapoler la majoration du lemme 2 aux valeurs $Q_{s, \underline{h}, \underline{j}}(\underline{\theta})$ pour tout $s \in \mathbb{N}, s<T$, avec $T$ vérifiant $T^{\prime}<T \leq c T^{\prime}$ où $c=c\left(y_{1}, \ldots, \bar{y}_{n}\right)$.

Soient $\underline{j} \in \mathcal{I}_{r}, \underline{\tilde{\theta}} \in B\left(\underline{\theta}, e^{-r}\right)$ tels que $\underline{j}=\underline{j}(\underline{\widetilde{\theta}})$ et $f$ la fonction entière définie par

$$
f(z)=\sum_{\substack{0 \leq \beta<L \\ 0 \leq \gamma<D}} D^{j} P_{\beta, \gamma}(\underline{\widetilde{\theta}}) \mathcal{M}_{\beta, \gamma}\left(z, e^{z}\right) .
$$

On a (cf. les définitions du 1er pas)

et

$$
\frac{d^{s}}{d z^{s}} f(z)=\sum_{\substack{0 \leq \beta<L \\ 0 \leq \gamma<D}} D^{j} P_{\beta, \gamma}(\underline{\widetilde{\theta}}) \Delta_{s} \mathcal{M}_{\beta, \gamma}\left(z, e^{z}\right)
$$

$$
\frac{d^{s}}{d z^{s}} f(\underline{h} \cdot \underline{y})=\sum_{\substack{0 \leq \beta<L \\ 0 \leq \gamma<D}} D^{\underline{j}} P_{\beta, \gamma}(\underline{\widetilde{\theta}}) \mathcal{M}_{\beta, \gamma, \underline{h}, s}(\underline{\theta}) .
$$


On a, de la même façon qu'au 1er pas, pour tout $s \in \mathbb{N}, 0 \leq s<T^{\prime}$, et tout $\underline{h} \in \mathbb{N}^{n},|\underline{h}|<M$,

$$
\begin{aligned}
\left|Q_{s, \underline{h}, \underline{j}}(\underline{\theta})-\frac{d^{s}}{d z^{s}} f(\underline{h} \cdot \underline{y})\right| \\
\quad \leq \sum_{\substack{0 \leq \beta<L \\
0 \leq \gamma<D}}\left|D^{j} P_{\beta, \gamma}(\underline{\widetilde{\theta}})-D^{j} P_{\beta, \gamma}(\underline{\theta})\right| \cdot\left|\mathcal{M}_{\beta, \gamma, \underline{h}, s}(\underline{\theta})\right| \\
\leq \exp \left(-r+2 \varphi_{2}\left(L, D, M, T^{\prime}\right)+\log L D\right) .
\end{aligned}
$$

Cette inégalité et le lemme 2 entraînent l'inégalité

$$
\max _{\substack{0 \leq s<T^{\prime} \\|\underline{h}|<M}}\left|\frac{d^{s}}{d z^{s}} f(\underline{h} \cdot \underline{y})\right| \leq \exp \left(-r+2 \varphi_{2}\left(L, D, M, T^{\prime}\right)+\log 4 L D\right) .
$$

D'autre part, la formule d'extrapolation (cf. [R], lemme 4.5) appliquée à $f$, avec $R_{1}>R_{2} \geq\left(\max _{1 \leq i \leq n} y_{i}+1\right) M$ et $\delta^{\prime}=\min _{|\underline{h}| \leq 2 M}\|\underline{h} \cdot \underline{y}\|$, montre que pour tout $s \in \mathbb{N}$, on a

$$
\begin{aligned}
& \frac{1}{s !}\left|\frac{d^{s}}{d z^{s}} f\right|_{R_{2}} \\
& \quad \leq 2|f|_{R_{1}}\left(\frac{4 R_{2}}{R_{1}}\right)^{T^{\prime} M^{n}}+\frac{2 M^{n}}{R_{2}}\left(\frac{33 R_{2}}{\delta^{\prime} M^{n / 2}}\right)^{T^{\prime} M^{n}} \max _{\substack{0 \leq s^{\prime}<T^{\prime} \\
|\underline{h}| \leq M}}\left|\frac{1}{s^{\prime} !} \frac{d^{s^{\prime}}}{d z^{s^{\prime}}} f(\underline{h} \cdot \underline{y})\right|
\end{aligned}
$$

où $|f|_{R}=\sup _{\|z\|=R}|f(z)|$.

Les nombres $y_{i}(1 \leq i \leq n)$ étant algébriques, l'inégalité de la taille (cf. $\left.\left[\mathrm{Wa}_{2}\right], 1.2 .4\right)$ montre que $\delta^{\prime} \geq \exp \left(-[\mathbb{K}: \mathbb{Q}] \log c_{6} M\right)$ où

$$
c_{6}=2 n \max _{1 \leq i \leq n}\left(\operatorname{den}\left(y_{i}\right)\right) \cdot \max _{1 \leq i \leq n}\left(\left|y_{i}\right|+1\right) .
$$

D'autre part, on a

$$
\begin{aligned}
|f|_{R_{1}} & \leq L D \max _{\substack{0 \leq \beta<L \\
0 \leq \gamma<D}}\left|D^{\underline{j}} P_{\beta, \gamma}(\underline{\tilde{\theta}})\right| R_{1}^{L} e^{R_{1} D} \\
& \leq \exp \left(L \log R_{1}+R_{1} D+\varphi_{2}\left(L, D, M, T^{\prime}\right)+\log L D\right) .
\end{aligned}
$$

En prenant $R_{1}=L, R_{2}=\left(\max _{1 \leq i \leq n}\left|y_{i}\right|+1\right) M$ et en supposant la condition

$$
L^{1 / 2}>4\left(\max _{1 \leq i \leq n}\left|y_{i}\right|+1\right) M
$$

vérifiée, on déduit de l'inégalité (5) et de la formule d'extrapolation précé- 
dente que pour tout $T \geq T^{\prime}$, on a

$$
\begin{aligned}
& \max _{\substack{0 \leq s<T \\
\|\underline{h}\|<M}}\left|\frac{d^{s} f}{d z^{s}}(\underline{h} \cdot \underline{y})\right| \\
& \leq \exp \left(L(\log L+D)+\varphi_{2}\left(L, D, M, T^{\prime}\right)+\log L D+T \log T-\frac{1}{2} T^{\prime} M^{n} \log L\right) \\
& \quad+\exp \left([\mathbb{K}: \mathbb{Q}] T^{\prime} M^{n} \log c_{7} M+2 \varphi_{2}\left(L, D, M, T^{\prime}\right)+\log 8 L D+T \log T-r\right)
\end{aligned}
$$

où

$$
c_{7}=c_{6}+33\left(\max _{1 \leq i \leq n}\left|y_{i}\right|+1\right)+n / 2-1 .
$$

Or, comme dans l'inégalité (4), pour tout $s \in \mathbb{N}, 0 \leq s<T$, et tout $\underline{h} \in \mathbb{N}^{n},|\underline{h}|<M$, on a

$$
\left|Q_{s, \underline{h}, \underline{j}}(\underline{\theta})-\frac{d^{s}}{d z^{s}} f(\underline{h} \cdot \underline{y})\right| \leq \exp \left(-r+\varphi_{2}(L, D, M, T)+\log L D\right),
$$

d'où

(6) $\left|Q_{s, h, \underline{j}}(\underline{\theta})\right|$

$$
\begin{aligned}
\leq & \exp \left(L(\log L+D)+\varphi_{2}\left(L, D, M, T^{\prime}\right)+\log L D+T \log T-\frac{1}{2} T^{\prime} M^{n} \log L\right) \\
& +\exp \left([\mathbb{K}: \mathbb{Q}] T^{\prime} M^{n} \log c_{7} M+2 \varphi_{2}\left(L, D, M, T^{\prime}\right)+\log 8 L D+T \log T-r\right) \\
& +\exp \left(-r+\varphi_{2}(L, D, M, T)+\log L D\right) .
\end{aligned}
$$

3e pas. Utilisation du lemme de zéros

Lemme 3. Soit $r$ un nombre réel tel que $r>\max _{1 \leq i \leq n}\left|y_{i}\right|$. Si les conditions

$$
T([M / 2])^{n}>4 L D
$$

et

$$
T>2 D
$$

sont vérifiées, alors les polynômes $Q_{s, \underline{h}, \underline{j}}\left(s \in \mathbb{N}, 0 \leq s<T, \underline{h} \in \mathbb{N}^{n},|\underline{h}|<\right.$ $\left.M, \underline{j} \in \mathcal{I}_{r}\right)$ n'ont pas de zéros communs dans la boule $B\left(\underline{\theta}, e^{-r}\right)$ de $\mathbb{C}^{n}$.

Preuve. Supposons que ces polynômes s'annulent en un point $\underline{\sigma}_{\widetilde{\theta}}=$ $\left(\widetilde{\theta}_{1}, \ldots, \widetilde{\theta}_{n}\right)$ de $B\left(\underline{\theta}, e^{-r}\right)$. Puisque $r>\max _{1 \leq i \leq n}\left|y_{i}\right|$, on en déduit que $\widetilde{\theta}_{i} \neq 0$ pour tout $i, i=1, \ldots, n$. Soit, alors, $\widetilde{y}_{i} \in \mathbb{C}$ tel que $e^{\tilde{y}_{i}}=\widetilde{\theta}_{i}(i=1, \ldots, n)$. Rappelons que, par définition, on a

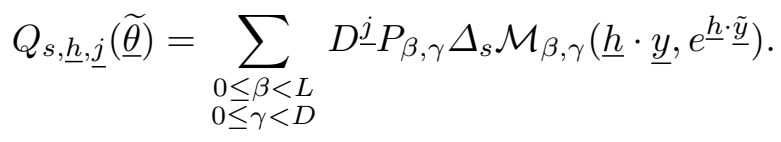


Considérons, en particulier, le $n$-uplet $\underline{j}$ de $\mathcal{I}_{r}$ tel que $\underline{j}=\underline{j}(\underline{\widetilde{\theta}})$ et le polynôme

$$
P(Z, X)=\sum_{\substack{0 \leq \beta<L \\ 0 \leq \gamma<D}} D^{j} P_{\beta, \gamma}(\underline{\widetilde{\theta}}) \mathcal{M}_{\beta, \gamma}(Z, X) .
$$

Pour $\underline{h} \in \mathbb{N}^{n},|\underline{h}|<M$, notons $F_{\underline{h}}$ la fonction entière définie par

$$
F_{\underline{h}}(z)=P\left(\underline{h} \cdot \underline{y}+z, e^{\underline{h} \cdot \underline{\tilde{y}}+z}\right) .
$$

On a

$$
\frac{d^{s}}{d z^{s}} F_{\underline{h}}(0)=Q_{s, \underline{h}, \underline{j}}(\underline{\widetilde{\theta}}) .
$$

Comme on a supposé que $Q_{s, \underline{h}, j}(\underline{\widetilde{\theta}})=0$, pour tout $s \in \mathbb{N}, 0 \leq s<T$, et tout $\underline{h} \in \mathbb{N}^{n},|\underline{h}|<M$, alors selon la définition de $\left[\mathrm{P}_{2}\right]$, le polynôme $P$ s'annule à l'ordre $T$ le long de l'application $\Psi$ définie par

$$
\Psi: \mathbb{C} \rightarrow \mathbf{G}_{a} \times \mathbf{G}_{m}, \quad z \rightarrow\left(z, e^{z}\right),
$$

sur l'ensemble $\widetilde{\Gamma}(M)=\left\{\left(\underline{h} \cdot \underline{y}, e^{\underline{h} \cdot \underline{\tilde{y}}}\right): \underline{h} \in \mathbb{N}^{n},|h|<M\right\}$.

Or $\underline{j}=: j(\underline{\widetilde{\theta}})$, donc les coefficients $D^{j} P_{\beta, \gamma}(\underline{\widetilde{\theta}}), 0 \leq \beta<L, 0 \leq \gamma<D$, de $P$ ne sont pas tous nuls, donc $P$ est non nul sur $\mathbf{G}_{a} \times \mathbf{G}_{m}$. Par suite le lemme de zéro (cf. th. 2.1 de $\left[\mathrm{P}_{2}\right]$ ) joint au lemme 3.4 de $\left[\mathrm{P}_{2}\right]$ montre qu'il existe un sous-groupe $G^{\prime}=G_{1}^{\prime} \times G_{2}^{\prime} \subsetneq \mathbf{G}_{a} \times \mathbf{G}_{m}$ de $\mathbf{G}_{a} \times \mathbf{G}_{m}$ tel que

$$
T \operatorname{card}\left(\left(\widetilde{\Gamma}(M / 2)+G^{\prime}\right) / G^{\prime}\right) \leq 2^{r_{1}+r_{2}} L^{r_{1}} D^{r_{2}}
$$

où $r_{1}=\operatorname{dim} \mathbf{G}_{a} / G_{1}^{\prime}$ et $r_{2}=\operatorname{dim} \mathbf{G}_{m} / G_{2}^{\prime}$.

Deux cas peuvent se présenter :

- ou bien $G_{1}^{\prime}=\mathbf{G}_{a}$, on a alors $r_{1}=0$ et l'inégalité (7) entraîne l'inégalité $T \leq 2 D$, ce qui est absurde vue la condition $\left(\mathcal{C}_{4}\right)$;

- ou bien $G_{1}^{\prime}=\{0\}$, on a alors $r_{1}=1$ et on a, dans ce cas,

$$
\operatorname{card}\left(\left(\widetilde{\Gamma}(M / 2)+G^{\prime}\right) / G^{\prime}\right) \geq([M / 2])^{n}
$$

et l'inégalité (7) entraîne l'inégalité $T([M / 2])^{n} \leq 4 L D$, ce qui contredit $\left(\mathcal{C}_{3}\right)$, d'où le lemme.

Choix des paramètres et preuve de la proposition principale. Soit $L_{0}=$ $L_{0}\left(y_{1}, \ldots, y_{n}\right)$ un nombre réel suffisamment grand; soient $L \geq L_{0}$ et $0<\nu$ $<1$ deux nombres réels vérifiant les conditions (1), (2) et (3) de la proposition principale. Posons

$$
\begin{gathered}
T^{\prime}=\left[4 L(\log L)^{-\nu}+1\right], \quad M=\left[(\log L)^{\nu / n}+1\right], \\
D=2^{n+5}[\mathbb{K}: \mathbb{Q}], \quad T=\left[2^{2 n+8}[\mathbb{K}: \mathbb{Q}] L(\log L)^{-\nu}\right], \quad r=L \log L .
\end{gathered}
$$

Les conditions $\left(\mathcal{C}_{1}\right),\left(\mathcal{C}_{2}\right),\left(\mathcal{C}_{3}\right)$ et $\left(\mathcal{C}_{4}\right)$ précédentes sont évidemment satisfaites avec ces paramètres. De plus, puisque $L \geq L_{0}$ et $(L, \nu)$ vérifie les 
inégalités (2) et (3) de la proposition principale, on en déduit que

$$
\varphi_{2}\left(L, D, M, T^{\prime}\right) \leq 9 L(\log L)^{1-\nu}
$$

et

$$
\varphi_{2}(L, D, M, T) \leq\left(2^{2 n+9}[\mathbb{K}: \mathbb{Q}]+1\right) L(\log L)^{1-\nu} .
$$

D'autre part, avec le choix ci-dessus des paramètres $D, M, T^{\prime}, T$ et puisque $(L, \nu)$ vérifie les inégalités $(2)$ et $(3)$ de la proposition principale, l'inégalité (6) précédente s'écrit

$$
\begin{aligned}
\left|Q_{s, \underline{h}, \underline{j}}(\underline{\theta})\right| \leq & \exp \left(-L \log L+\left(2^{2 n+9}[\mathbb{K}: \mathbb{Q}]+11\right) L(\log L)^{1-\nu}\right) \\
& +\exp \left(-L \log L+\left(21+2^{2 n+9}[\mathbb{K}: \mathbb{Q}]\right) L(\log L)^{1-\nu}\right) \\
& +\exp \left(-L \log L+\left(2^{2 n+9}[\mathbb{K}: \mathbb{Q}]+2\right) L(\log L)^{1-\nu}\right)
\end{aligned}
$$

et par suite l'inégalité (1) de la proposition principale entraîne

$$
\left|Q_{s, \underline{h}, \underline{j}}(\underline{\theta})\right| \leq \exp \left(-\frac{1}{2} L \log L\right)
$$

ou encore

$$
\left|Q_{s, \underline{h}, \underline{j}}(\underline{\theta})\right| \leq \exp \left(-r_{1 / 2}(L)\right)
$$

Par ailleurs, on a

$$
\begin{aligned}
\operatorname{deg} Q_{s, \underline{h}, \underline{j}} & \leq \max _{\beta, \gamma}\left(\operatorname{deg} D_{-}^{j} P_{\beta, \gamma}+\operatorname{deg} \mathcal{M}_{\beta, \gamma, s, \underline{h}}\right) \\
& \leq 2 n D M \leq 2^{n+7}[\mathbb{K}: \mathbb{Q}] n(\log L)^{\nu / n} ;
\end{aligned}
$$

avec les notations du chapitre I, cette inégalité s'écrit

$$
\operatorname{deg} Q_{s, \underline{h}, \underline{j}} \leq \delta_{c_{4}}(L, \nu) \quad \text { avec } \quad c_{4}=2^{n+7}[\mathbb{K}: \mathbb{Q}] n .
$$

On a aussi

$$
\begin{aligned}
h\left(Q_{s, \underline{h}, \underline{j}}\right) & \leq \max _{\beta, \gamma}\left(h\left(D^{\underline{j}} P_{\beta, \gamma}\right)+h\left(\mathcal{M}_{\beta, \gamma, s, \underline{h}}\right)\right)+2 n D M \\
& \leq 2 \varphi_{1}(L, D, M, T)+2 n D M \leq 2 \varphi_{2}(L, D, M, T) \\
& \leq\left(2^{2 n+10}[\mathbb{K}: \mathbb{Q}]+2\right) L(\log L)^{1-\nu},
\end{aligned}
$$

d'où

$$
h\left(Q_{s, \underline{h}, \underline{j}}\right) \leq H_{c_{5}}(L, \nu) \quad \text { avec } \quad c_{5}=2^{2 n+10}[\mathbb{K}: \mathbb{Q}]+2 .
$$

Soit $I_{L, \nu}$ l'idéal de $\mathbb{K}\left[Y_{1}, \ldots, Y_{n}\right]$ engendré par la famille des polynômes $Q_{s, \underline{h}, j}\left(Y_{1}, \ldots, Y_{n}\right), s \in \mathbb{N}, 0 \leq s<T, \underline{h} \in \mathbb{N}^{n},|\underline{h}|<M, \underline{j} \in \mathcal{I}_{r}$, où $T, \bar{M}, r$ sont les paramètres définis ci-dessus en fonction de $L$ et $\nu$. D'après le lemme 3 , l'idéal $I_{L, \nu}$ n'a pas de zéros dans la boule de $\mathbb{C}^{n}$ de centre $\underline{\theta}$ et de rayon $\exp (-r(L))$; il vérifie donc la condition (i) de la proposition principale. L'inégalité (8) (resp. (9) et (10)) montre que $I_{L, \nu}$ vérifie la condition (ii) (resp. (iii)) de la proposition principale. 
III. Preuve du théorème. Rappelons que $y_{1}, \ldots, y_{n}$ désignent des nombres algébriques $\mathbb{Q}$-linéairement indépendants et on note

$$
\mathbb{K}=\mathbb{Q}\left(y_{1}, \ldots, y_{n}\right) \quad \text { et } \quad \theta_{i}=e^{y_{i}}, \quad 1 \leq i \leq n .
$$

Pour $f$ et $g$ deux fonctions de $\mathbb{R}^{+}$dans $\mathbb{R}^{+}$, on note $f \gg g$ s'il existe $c^{\prime}=c^{\prime}\left(y_{1}, \ldots, y_{n}\right)>0$ tel que pour tout $L \geq L_{0}$ on ait $f(L) \geq c^{\prime} g(L)$ et $f \gg \ll g \Leftrightarrow f \ll g$ et $g \ll f$.

Soit $C=C\left(y_{1}, \ldots, y_{n}\right)$ un nombre réel suffisamment grand par rapport aux constantes $c_{1}, \ldots, c_{i}, \ldots$ qui interviennent dans ce texte. Soient $h, D$ deux nombres réels $>0$. Posons

$$
\begin{aligned}
h_{1} & =\left(h+\exp \left(C D^{n} \log (D+1)\right)\right), \\
\nu & =\left(n \log D+\log c_{8}\right) / \log \log h_{1},
\end{aligned}
$$

où

$$
c_{8}=2^{n\left(4 n^{2}+16 n+12\right)} n^{n^{2}+2}([\mathbb{K}: \mathbb{Q}])^{n^{2}+1}(4[\mathbb{K}: \mathbb{Q}]+n+1)^{n+1} .
$$

On a bien $0<\nu<1$.

Rappelons les notations $H_{1}(L, \nu)=L(\log L)^{1-\nu}, \delta_{1}(L, \nu)=(\log L)^{\nu / n}$ et $r_{1}(L)=L \log L$.

Soit $L$ le plus petit entier vérifiant

$$
c_{9} h_{1} D^{n} \leq r_{1}(L),
$$

où

$$
c_{9}=2^{n\left(4 n^{2}+18 n+25\right)+3} n^{n^{2}+n+2}([\mathbb{K}: \mathbb{Q}])^{n^{2}+n+1}(4[\mathbb{K}: \mathbb{Q}]+n+1)^{n+2} .
$$

Posons $\delta=\delta_{c_{4}}(L, \nu), \tau=H_{c_{5}}(L, \nu)+\delta(n+1) \log (n+1), \sigma=2, U=\frac{1}{2} r_{1}(L)$ où $c_{4}$ et $c_{5}$ sont les constantes définies dans la proposition principale.

Nous allons montrer que ces paramètres vérifient les conditions du critère du chapitre I.

L'inégalité (11) et $L$ suffisamment grand entraînent que $\log \log L \geq$ $\frac{1}{2} \log \log h_{1}$, et de la définition de $\nu$, on tire que $\log \log h_{1} \geq\left(\log c_{8}\right) / \nu$, d'où $\log \log L \geq\left(\log c_{8}\right) / 2 \nu$ et comme $c_{8}>2^{6} c_{5}^{2}$, on en déduit que $U \geq$ $2 \max \left(\tau, \sigma^{n}\right)$ et $\delta>1$.

Soit $S$ un entier vérifiant la condition (a) $\tau / \sigma^{n}<S \leq U / \sigma^{n}$ du critère, et $L^{\prime}$ le nombre réel défini par $r_{1}\left(L^{\prime}\right)=S \sigma^{n+1}$. Montrons que $\left(L^{\prime}, \nu\right)$ vérifie les hypothèses de la proposition principale.

Comme $S>\tau / \sigma^{n}$, on a $r_{1}\left(L^{\prime}\right)>\tau$ et, par suite, $\log L^{\prime} \gg \log L$; or l'inégalité (11) entraîne $\log L \gg \log h_{1}$, d'où $\log L^{\prime} \gg \log h_{1}$ et puisque $\log h_{1}>C$ et que $C$ est suffisamment grand par rapport à $L_{0}$, on en déduit que $L^{\prime}>L_{0}$.

Par ailleurs, $\log L^{\prime} \gg \log h_{1}$ et $\log h_{1}>C$ entraînent l'inégalité $\log \log L^{\prime}$ $\geq \frac{1}{2} \log \log h_{1} ;$ or $\log \log h_{1} \geq\left(\log c_{8}\right) / \nu$ et $\log c_{8}>4(n+7) \log 2[\mathbb{K}: \mathbb{Q}]$, 
d'où

$$
\log \log L^{\prime} \geq \frac{2(n+7) \log 2[\mathbb{K}: \mathbb{Q}]}{\nu} ;
$$

ainsi $\left(L^{\prime}, \nu\right)$ vérifie l'inégalité (1) de la proposition principale.

D'autre part,

$$
\left(\log L^{\prime}\right)^{1-\nu} \gg\left(\log h_{1}\right)^{1-\nu} \quad \text { et } \quad\left(\log h_{1}\right)^{1-\nu}=\frac{\log h_{1}}{c_{8} D^{n}} \geq \frac{C}{c_{8}} ;
$$

comme $C$ est suffisamment grand, on en déduit que $\left(\log L^{\prime}\right)^{1-\nu} \geq c_{3}$; ainsi $\left(L^{\prime}, \nu\right)$ vérifie la condition (2) de la proposition principale.

Remarquons que $S \leq U / \sigma^{n}$ entraîne $r_{1}\left(L^{\prime}\right)<U \sigma \leq r_{1}(L)$ et, par suite, $L^{\prime} \leq L$. On a alors

$$
\nu\left(\log L^{\prime}\right)^{\nu} \log \log L^{\prime} \leq \nu(\log L)^{\nu} \log \log L .
$$

Or, $L-1$ ne vérifie pas l'inégalité (11), d'où $\log L \ll \log h_{1}$ et, par suite,

$$
\nu\left(\log L^{\prime}\right)^{\nu} \log \log L^{\prime} \ll \nu\left(\log h_{1}\right) \log \log h_{1} ;
$$

de la définition de $\nu$ et $h_{1}$, on tire les inégalités

$$
\nu\left(\log h_{1}\right)^{\nu} \log \log h_{1} \leq c_{8} D^{n} \log c_{8} D^{n} \leq \frac{c_{8}\left(n+\log c_{8}\right) \log h_{1}}{C} .
$$

Comme $C$ est suffisamment grand et que $\log h_{1} \ll \log L^{\prime}$, on en déduit que

$$
8[\mathbb{K}: \mathbb{Q}] \nu\left(\log L^{\prime}\right)^{\nu} \log \log L^{\prime} \leq \log L^{\prime} ;
$$

la condition (3) de la proposition principale est donc vérifiée par $\left(L^{\prime}, \nu\right)$. Alors, d'après cette même proposition, il existe un idéal $I_{L^{\prime}, \nu}=:\left(G_{L^{\prime}, \nu, j}\right.$ : $\left.1 \leq j \leq m^{\prime}\left(L^{\prime}, \nu\right)\right)$ de $\mathbb{K}\left[X_{1}, \ldots, X_{n}\right]$ vérifiant les propriétés (i), (ii) et (iii).

Posons $\left\{Q_{s, i}: 1 \leq i \leq m^{\prime}(S)\right\}=\left\{G_{L^{\prime}, \nu, j}: 1 \leq j \leq m^{\prime}\left(L^{\prime}, \nu\right)\right\}$.

Les propriétés (i), (ii) et (iii) de la proposition principale montrent que les propriétés (e), (d), (c) et (b) du critère du chapitre I sont satisfaites avec les paramètres $\delta, \sigma, \tau$ et $U$ définis ci-dessus et avec $\mathbb{K}=\mathbb{Q}\left(y_{1}, \ldots, y_{n}\right)$ et $\underline{\theta}=\left(e^{y_{1}}, \ldots, e^{y_{n}}\right)$.

La propriété (f) de ce critère est vérifiée avec des paramètres $h, D, \delta, \sigma$, $\tau$ et $U$. En effet, d'après (11), on a

$$
h_{1} \delta^{n} \leq c_{9}^{-1} r_{1}(L) D^{-n} \delta^{n} .
$$

Or, de la définition de $\nu$, on tire l'égalité $D=\left(c_{8}^{-1}\left(\log h_{1}\right)^{\nu}\right)^{1 / n}$. Comme $L-1$ ne vérifie par (11) et que $h_{1}$ suffisamment grand, on a $\log h_{1} \geq \frac{1}{2} \log L$, d'où

$$
h_{1} \delta^{n} \leq c_{9}^{-1} c_{8} 2^{\nu} r_{1}(L)(\log L)^{-\nu} \delta^{n} \leq 2^{\nu} c_{9}^{-1} c_{8} c_{4}^{n} r_{1}(L) .
$$

Comme $\nu<1$, on a

$$
h_{1} \delta^{n} \leq 2 c_{9}^{-1} c_{8} c_{4}^{n} r_{1}(L)
$$


D'autre part, on a

$$
\begin{aligned}
n \tau D \delta^{n-1}+ & (n+1)^{2} \log (n+1) D \delta^{n} \\
\leq & n H_{c_{5}}(L, \nu) D \delta^{n-1}+(n+1)(2 n+1) \log (n+1) D \delta^{n} \\
\leq & n c_{5} c_{4}^{n-1} c_{8}^{-1 / n}\left(\log h_{1}\right)^{\nu / n} L(\log L)^{1-\nu}(\log L)^{\nu(n-1) / n} \\
& +(n+1)(2 n+1) \log (n+1) c_{8}^{-1 / n} c_{4}^{n}\left(\log h_{1}\right)^{\nu / n}(\log L)^{\nu} .
\end{aligned}
$$

Or, d'après (11), on a $\log h_{1} \leq 2 \log L$, d'où

$n \tau D \delta^{n-1}+(n+1)^{2} \log (n+1) D \delta^{n}$

$\leq 2 n c_{5} c_{4}^{n-1} c_{8}^{-1 / n} L(\log L)+2(n+1)(2 n+1) \log (n+1) c_{8}^{-1 / n} c_{4}^{n}(\log L)^{\nu(1+1 / n)}$.

Comme $L$ est assez grand, on en déduit

$$
n \tau D \delta^{n-1}+(n+1)^{2} \log (n+1) D \delta^{n} \leq\left(2 n c_{5} c_{4}^{n-1}+1\right) c_{8}^{-1 / n} r_{1}(L) .
$$

Puisque

$$
c_{8}^{1 / n} \geq 4\left(2 n c_{5} c_{4}^{n-1}+1\right)(4[\mathbb{K}: \mathbb{Q}]+n+1)(27 \sigma)^{n}
$$

et

$$
c_{9} \geq 8 c_{8} c_{4}^{n}(4[\mathbb{K}: \mathbb{Q}]+n+1)(27 \sigma)^{n},
$$

les inégalités (12) et (13) entraînent que

$$
\begin{array}{r}
(4[\mathbb{K}: \mathbb{Q}]+n+1)(27 \sigma)^{n}\left(h_{1} \delta^{n}+\left(n \tau+\delta(n+1)^{2} \log (n+1)\right) D \delta^{n+1}\right) \\
\leq \frac{1}{2} r_{1}(L) \leq U,
\end{array}
$$

donc la propriété (f) du critère est vérifiée.

Remarquons que, puisque $L$ est assez grand, on a $U \leq r_{1}(L-1)$ et comme $L-1$ ne vérifie pas $(11)$, on a $r_{1}(L-1)<c_{9} h_{1} D^{n}$ et par suite $U<c_{9} h_{1} D^{n}$.

Le critère du chapitre I montre alors que pour tout polynôme non nul $P$ de $\mathbb{Z}\left[X_{1}, \ldots, X_{n}\right]$, de degré $\leq D$ et de hauteur logarithmique $\leq h$, on a

$$
\log \left|P\left(\theta_{1}, \ldots, \theta_{n}\right)\right| \geq-U-n \log 2(n+1) D-2 n \log (n+1)-n^{2} \log |\underline{\theta}| .
$$

Or $C$ est suffisamment grand par rapport à $n$ et $|\underline{\theta}|$; on en déduit que

$$
n \log 2(n+1) D+2 n \log (n+1)+n^{2} \log |\underline{\theta}| \leq h_{1}
$$

et, comme $U<c_{9} h_{1} D^{n}$, on a $\log \left|P\left(\theta_{1}, \ldots, \theta_{n}\right)\right| \geq-2 c_{9} h_{1} D^{n}$, d'où le théorème.

$\mathrm{Au}$ cours de la démonstration, les constantes notées $c_{1}, \ldots, c_{i}, \ldots$ ont été explicitées pour avoir des énoncés plus précis; mais ces constantes ne sont pas toujours les meilleures possibles puisque nous les avons majorées grossièrement pour les rendre plus présentables.

Signalons que cette démonstration peut être adaptée pour retrouver l'analogue elliptique du théorème principal de ce texte, résultat démontré par Y. Nesterenko dans $\left[\mathrm{N}_{3}\right]$. 
Je tiens à remercier P. Philippon pour ses commentaires sur une première version de ce texte. Je remercie aussi le referee pour ses remarques qui m'ont permis d'améliorer la présentation.

\section{Références}

[D] G. Diaz, Grands degrés de transcendance pour des familles d'exponentielles, J. Number Theory 31 (1989), 1-23.

[J] E. M. Jabbouri, Sur un critère d'indépendance algébrique de P. Philippon, dans : Approximations diophantiennes et nombres transcendants, Luminy 1990, P. Philippon (éd.), Walter de Gruyter, 1992, 195-202.

[M] K. Mahler, Zur Approximation der Exponential Funktion und des Logarithmus I, J. Reine Angew. Math. 166 (1932), 118-136.

$\left[\mathrm{N}_{1}\right]$ Yu. V. Nesterenko, Estimates for the orders of zeros of functions of a certain class and their applications in the theory of transcendental numbers, Izv. Akad. Nauk SSSR Ser. Mat. 41 (1977), 253-284 (en russe); trad. anglaise: Math. USSRIzv. 11 (1977).

$\left[\mathrm{N}_{2}\right] \quad-$, On algebraic independence of algebraic powers of algebraic numbers, Mat. Sb. 123 (165) (1984), 435-459 (en russe); trad. anglaise: Math. USSR-Sb. 51 (1985).

$\left[\mathrm{N}_{3}\right]-$, On a measure of algebraic independence of the values of elliptic functions, dans : Approximations diophantiennes et nombres transcendants, Luminy 1990, P. Philippon (éd.), Walter de Gruyter, 1992, 239-248.

$\left[\mathrm{P}_{1}\right] \quad \mathrm{P}$. Philippon, Critères pour l'indépendance algébrique, Inst. Hautes Études Sci. Publ. Math. 64 (1986), 5-52.

$\left[\mathrm{P}_{2}\right] \quad-$, Lemmes de zéros dans les groupes algébriques commutatifs, Bull. Soc. Math. France 114 (1986), 335-383.

[R] E. Reyssat, Approximation algébrique de nombres liés aux fonctions elliptiques, ibid. 108 (1980), 47-79.

[S] C. Siegel, Transcendental Numbers, Ann. of Math. Stud., Princeton, 1949.

[T] T. Töpfer, Über lineare Unabhängigkeit in algebraischen Zahlkörpern, à paraître.

[Wa 1 M. Waldschmidt, Nombres transcendants et groupes algébriques, Astérisque 69-70 (1979).

[Wa $\left.\mathrm{W}_{2}\right] \quad-$, Nombres transcendants, Lecture Notes in Math. 402, Springer, 1974.

U.F.R. DE MATHÉMATIQUES

UNIVERSITÉ DES SCIENCES ET TECHNOLOGIES DE LILLE

59655 VILLENEUVE D'ASCQ CEDEX, FRANCE 